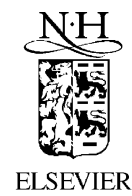

\title{
Coalition structure values in differential information economies: is unity a strength?
}

\author{
Stefan Krasa ${ }^{\mathrm{a}}$, Akram Temimi $^{\mathrm{b}}$, Nicholas C. Yannelis ${ }^{\mathrm{a}, *}$ \\ a Department of Economics, University of Illinois at Urbana-Champaign, \\ 1206 South Sixth Street, Champaign, IL 61820, USA \\ b Department of Economics, Finance and Legal Studies, University of Alabama, Tuscaloosa, AL 35487, USA
}

Received 30 January 2002; received in revised form 28 June 2002; accepted 3 July 2002

\begin{abstract}
The coalition structure (CS) value, introduced by Owen [G. Owen, Values of games with a priori unions, in: Essays in Mathematical Economics and Game Theory, Springer Verlag, Berlin, 1977] and Hart and Kurz [Econometrica 51 (1983) 1047], generalizes the Shapley value to social situations where coalitions form for the purpose of bargaining. This paper introduces the CS value to economies with differential information. We show that the private CS values exists and is Bayesian incentive compatible. Moreover, we construct examples that go against the intuitive viewpoint that "unity is strength". In particular, we consider a three-person economy in which two agents bargain as a unit against the third agent. We show that bargaining as a unit is advantageous if and only if information is complete. This result sheds new light on bargaining under differential information. (C) 2002 Elsevier Science B.V. All rights reserved.
\end{abstract}

Keywords: Coalition structure value; Differential information economies; Bargaining; Under differential information

\section{Introduction}

Recent work on differential information economies has indicated that cooperative solution concepts, such as the core, the Shapley value, and the bargaining set provide successful alternatives to the rational expectations equilibrium (see Allen and Yannelis, 2001 and the references therein). In particular, as first shown in Krasa and Yannelis (1994), the Shapley value is sensitive to information asymmetries and rewards agents with superior information, features that are not shared by the traditional rational expectations equilibrium.

In this paper, we pursue this line of research further, going beyond Krasa and Yannelis (1994, 1996), by introducing differential information into the coalition structure (CS) value

\footnotetext{
* Corresponding author. Tel.: +1-217-333-0120; fax: +1-217-244-6678.
} 
concept of Owen (1977) and Hart and Kurz (1983). One of the main properties of the CS value is that if agents are allowed to form coalitions to bargain as a unit, they may do so to strengthen their situation, that is, to increase their payoffs. The intent of the coalition structure value is to take into account situations where groups of players organize themselves for the purpose of bargaining with the rest of the players. Examples include political parties, unions, and cartels. In particular, Hart and Kurz (1983) construct a three-agent economy, in which the first two agents obtain higher payoffs whenever they bargain as a unit against the third agent, compared to the standard Shapley value that allows arbitrary coalition formation.

Given the appealing features of the CS value, it is of interest to know how this concept behaves in differential information economies. To this end we introduce differential information into the CS value concept. In particular, we consider two new equilibrium concepts: The private CS value and the Bayesian incentive compatible (BIC) CS value. In general, the two concepts differ, but we show that: (i) private CS values are always incentive compatible; and (ii) the set of attainable utilities of the transferable utility (TU) game derived from an economy with differential information is always a subset of the set of BIC attainable utilities. It turns out that the private $\mathrm{CS}$ value always exists, but the BIC value need not exist because the set of all BIC allocations need not be a convex set.

What we found most surprising, however, is that the intuitive statement that "unity is strength" ceases to be true for the CS value once differential information is introduced. In particular, we construct a three-person economy in which two agents bargain as a unit against the third agent. We show that bargaining as a unit is advantageous if and only if information is complete.

The paper proceeds as follows. In Section 2 we introduce the economy with differential information. In Section 3 we show two alternative ways how a TU game can be derived from an economy with differential information, and a comparison is provided. In Section 4 we introduce the main concepts of this paper, the coalition structure values of a differential information economy. Section 5 shows that coalitional bargaining may not be advantageous if informational asymmetries matter. All proofs are in Appendix A.

\section{The economy with differential information}

We consider an exchange economy that extends over two time periods, $t=0$, where consumption takes place in $t=1$. At $t=0$ there is uncertainty over the state of nature described by a probability space $(\Omega, \mathcal{F}, \mu)$. Agents are indexed by $i \in I=\{1, \ldots, n\}$.

In each state $\omega$ there are $\ell$ goods. The commodity space is therefore $\mathbb{R}_{+}^{\ell}$. Each agent $i$ 's endowment is given by $e_{i}: \Omega \rightarrow \mathbb{R}_{+}^{\ell}$.

At $t=0$ agents will agree on net-trades that may be contingent on the state of nature at $t=1$. However, agents are differentially informed with respect to the true state of nature. Specifically, we assume that at $t=1$ agents do not necessarily know which state $\omega \in \Omega$ has actually occurred. They know their own endowment realization, and every agent $i$ might have some additional information about the state described by a $\sigma$-algebra $\mathcal{F}_{i}$ with $\mathcal{F}_{i} \subset \mathcal{F}$. We assume that $\mathcal{F}_{i}$ is generated by a countable partition of $\Omega$. With a slight abuse of notation we will write $\mathcal{F}_{i}$ both for agent $i$ 's $\sigma$-algebra and for agent $i$ 's partition. By assumption, 
agents can always observe their own endowment realization, i.e. $e_{i}$ is $\mathcal{F}_{i}$-measurable for each agent $i \in I$.

In summary, an exchange economy with differential information is given by

$$
\mathcal{E}=\left\{\left(X_{i}, u_{i}, \mathcal{F}_{i}, e_{i}, \mu\right): i=1, \ldots, n\right\},
$$

where:

1. $X_{i}(\omega)=\mathbb{R}_{+}^{\ell}$, for all $\omega \in \Omega$, is agent $i$ 's consumption set;

2. $u_{i}: \Omega \times \mathbb{R}_{+}^{\ell} \rightarrow \mathbb{R}$ is agent $i$ 's utility function;

3. $\mathcal{F}_{i}$ is a measurable countable partition of $\Omega$, denoting the private information of agent $i$;

4. $e_{i}: \Omega \rightarrow \mathbb{R}_{+}^{\ell}$ is agent $i$ 's initial endowment, where each $e_{i}$ is $\mathcal{F}_{i}$-measurable and integrable;

5. $\mu$ is the probability measure on $\Omega$ denoting the common prior of all agents.

Agent $i$ 's ex-ante expected utility of consuming $x_{i}: \Omega \rightarrow \mathbb{R}_{+}^{\ell}$ is

$$
\int_{\Omega} u_{i}\left(\omega, x_{i}(\omega)\right) \mathrm{d} \mu(\omega)
$$

Let $\omega^{*}$ be the true state of the economy in $t=1$. Then at the interim, agent $i$ observes the event $E_{i}\left(\omega^{*}\right)$ in the partition $\mathcal{F}_{i}$ which contains $\omega^{*}$. Agent $i$ 's updated prior is then given by $\mu\left(\cdot \mid E_{i}\left(\omega^{*}\right)\right)$. $^{1}$

An allocation will be denoted by $\left(x_{i}\right)_{i \in I}$. An allocation is feasible if $\sum_{i \in I} x_{i}(\omega)=$ $\sum_{i \in I} e_{i}(\omega), \mu$-a.e.

Throughout the paper, we assume for each $\omega \in \Omega$ that the utility function $u_{i}(\omega, \cdot)$ of each agent $i$ is continuous and concave.

\section{The TU game}

As in the definition of the standard value allocation concept, we must first derive a transferable utility (TU) game in which each agent's utility is weighted by a factor $\lambda_{i}$, $(i=1, \ldots, n)$, which allows interpersonal utility comparisons. In the value allocation itself no side-payments are necessary. A game with side-payments is then defined as follows.

Definition 1. A game with side-payments $(I, V)$ consists of a finite set of agents $I=$ $\{1, \ldots, n\}$ and a superadditive, real valued function $V$ defined on $2^{I}$ such that $V(\emptyset)=0$. Each $S \subset I$ is called a coalition and $V(S)$ is the "worth" of coalition $S$.

We now define for each economy with differential information, $\mathcal{E}$, and for each set of weights, $\left\{\lambda_{i}: i=1, \ldots, n\right\}$, the associated game with side-payments. Clearly, each coalition of agents $S$ can obtain only those allocations that are feasible for the coalition, i.e. $\sum_{i \in S} x_{i}(\omega)=\sum_{i \in S} e_{i}(\omega), \mu$-a.e. However, trade among agents is also restricted because of private information. We consider two alternative ways to specify the set of allocations that

\footnotetext{
${ }^{1}$ Thus, $\mu\left(A \mid E_{i}\left(\omega^{*}\right)\right)=\mu\left(A \cap E_{i}\left(\omega^{*}\right)\right) / \mu\left(E_{i}\left(\omega^{*}\right)\right)$ for any $A \in \mathcal{F}$, where $\mu\left(E_{i}\left(\omega^{*}\right)\right)>0$.
} 
a coalition $S$ can obtain. The first way is to consider trades, which are private information measurable. That is, agents can only make net trades based on their own information. The second way allows for trades, which are Bayesian incentive compatible. The relationship between these two alternative scenarios is shown in Theorem 1. More formally,

1. In the first specification, we assume that each coalition of agents, $S$, can achieve any feasible allocation $\left(x_{i}\right)_{i \in S}$ that fulfills private measurability, i.e. $x_{i}$ is $\mathcal{F}_{i}$-measurable for all agents $i$ in $S$.

2. Alternatively, we assume that each coalition can obtain any feasible allocation $\left(x_{i}\right)_{i \in S}$ that is Bayesian incentive compatible (BIC) for coalition $S$ and $\bigvee_{i \in S} \mathcal{F}_{i}$-measurable.

Before deriving the TU game for the differential information economy, we define Bayesian incentive compatibility (see Glycopantis et al., 2001 for a similar definition).

Definition 2. An allocation $x=\left(x_{i}\right)_{i \in S}$ is Bayesian incentive compatible (BIC) for coalition $S$ if and only if there does not exist an agent $j \in S$ and states $\omega^{*}, \omega^{\prime} \in \Omega$ such that

$$
\begin{gathered}
\int_{Z_{S}\left(\omega^{*}\right)} u_{j}\left(\omega, e_{j}(\omega)+x_{j}\left(\omega^{\prime}\right)-e_{j}\left(\omega^{\prime}\right)\right) \mathrm{d} \mu\left(\omega \mid E_{j}\left(\omega^{*}\right)\right) \\
>\int_{Z_{S}\left(\omega^{*}\right)} u_{j}\left(\omega, x_{j}(\omega)\right) \mathrm{d} \mu\left(\omega \mid E_{j}\left(\omega^{*}\right)\right)
\end{gathered}
$$

where $Z_{S}\left(\omega^{*}\right)=\bigcap_{i \in S} E_{i}\left(\omega^{*}\right), \omega^{\prime} \in \bigcap_{i \in S \backslash\{j\}} E_{i}\left(\omega^{*}\right)$ and $e_{j}\left(\omega^{*}\right)+x_{j}\left(\omega^{\prime}\right)-e_{j}\left(\omega^{\prime}\right) \in \mathbb{R}_{+}^{\ell}$.

Inequality (1) states that agents cannot improve by misreporting their information. Definition 1 also requires that agent $j$ 's false report cannot always be detected by other agents in the coalition. In particular, in order for the false report report $\omega^{\prime}$ not to be detected when $\omega^{*}$ is the true state, $\omega^{\prime} \in \bigcap_{i \in S \backslash\{j\}} E_{i}\left(\omega^{*}\right)$ must hold. Moreover, note that if (1) holds then $\mu\left(Z_{S}\left(\omega^{*}\right) \mid E_{j}\left(\omega^{*}\right)\right)>0$. Thus, if agent $j$ can improve by a misrepresenting his information, then his false report should not be detectable with certainty (i.e. the set of states $\omega \neq \omega^{\prime}$ that are consistent with $\omega^{\prime}$ has positive measure). ${ }^{2}$

The reader might wonder why we do not allow misreports that can be detected by other agents with certainty. In particular, we interpret the example in Section 5 as a model in which agents sign contracts ex-ante to insure each other against low endowment realizations. If agent $i$ receives a high realization he must make a payment to agents who received a lower realization. Now assume that agent $j$ makes a report which is inconsistent with those of the other agents. If such a report leads to no trade, then agent $j$ can keep his endowment, effectively reneging on his ex-ante agreement to insure the other agents. Therefore, in order to make insurance contracts enforceable, we must assume that agents cannot make reports that are inconsistent.

\footnotetext{
${ }^{2}$ In order to see what consistency of reports means in a simple example, assume that $\Omega=\{a, b, c\}$, that there are two agents $I=\{1,2\}$ and $\mathcal{F}_{1}=\{\{a, b\},\{c\}\}, \mathcal{F}_{2}=\{\{a, c\},\{b\}\}$. Assume for example that agent 1 reports $c$ and agent 2 reports $b$. Then these reports are not consistent because $\{c\} \cap\{b\}=\emptyset$. In contrast, the report $\{a, b\}$ by agent 1 and $\{b\}$ by agent 2 is consistent.
} 
We are now ready to define the two alternative versions of the TU game. First, for every coalition $S \subset I$ let

$$
V_{\lambda}^{p}(S)=\max _{\left(x_{i}\right)_{i \in S}} \sum_{i \in S} \lambda_{i} \int u_{i}\left(x_{i}(\omega)\right) \mathrm{d} \mu(\omega)
$$

s.t.

(i) $\sum_{i \in S} x_{i}(\omega)=\sum_{i \in S} e_{i}(\omega), \mu$-a.e.

(ii) $x_{i}$ is $\mathcal{F}_{i}$-measurable, $\forall i \in S$.

Second, we define

$$
V_{\lambda}^{\mathrm{BIC}}(S)=\max _{\left(x_{i}\right)_{i \in S}} \sum_{i \in S} \lambda_{i} \int u_{i}\left(x_{i}(\omega)\right) \mathrm{d} \mu(\omega),
$$

s.t.

(i) $\sum_{i \in S} x_{i}(\omega)=\sum_{i \in S} e_{i}(\omega)$, $\mu$-a.e.

(ii) $x_{i}$ is $\bigvee_{i \in S} \mathcal{F}_{i}$-measurable, $\forall i \in S$.

(iii) $\left(x_{i}\right)_{i \in S}$ is BIC for coalition $S$.

Note that (ii) in (3) is needed to ensure that agents can obtain allocation $\left(x_{i}\right)_{i \in S}$, given the available information.

We characterize the relationship between the two TU games in Theorem 1 below. The proof is in Appendix A.

Theorem 1. Assume that $\lambda_{i}>0$ for all $i \in I$. Then

1. $V_{\lambda}^{p}(S) \leq V_{\lambda}^{\mathrm{BIC}}(S)$ for all $S \subset I$.

2. Consider a feasible allocation $\left(x_{i}\right)_{i \in S}$ for coalition $S$ that is measurable with respect to private information, i.e. $x_{i}$ is $\mathcal{F}_{i}$-measurable for all $i \in S$. Then $\left(x_{i}\right)_{i \in S}$ is BIC for coalition $S$.

Clearly, the private and the BIC value allocation differ when $V_{\lambda}^{p}(I)<V_{\lambda}^{\mathrm{BIC}}(I)$.

\section{The coalition structure $(\mathrm{CS})$ value allocation}

The standard Shapley value of the game (I, V), (Shapley, 1953), is a rule that assigns to each agent $i$ a payoff $\mathrm{Sh}_{i}$, given by the formula

$$
\mathrm{Sh}_{i}=\sum_{S \subset I, S \supset\{i\}} \frac{(|S|-1) !(|I|-|S|) !}{|I| !}[V(S)-V(S \backslash\{i\})] .
$$

Following the treatment in Hart and Kurz (1983), we first generalize the Shapley value formula to account for coalition formation (see also Owen, 1977).

Let $\mathcal{B}=\left\{B_{1}, \ldots, B_{m}\right\}$ be a partition of the set of agents $I$. We refer to $\mathcal{B}$ as a coalition structure. As in the standard Shapley value, we wish to measure each agent's expected contribution to a coalition that he/she is a member of. However, this expected contribution should be compatible with the coalition structure $\mathcal{B}$. 
Consider all possible ways to order the set of coalitions in $\mathcal{B}$ and the agents within each coalition. We say that a complete linear order on $I$ is consistent with $\mathcal{B}$ if, for all $k=1, \ldots, m$ and all $i, j$ in $B_{k}$, all elements of $I$ between $i$ and $j$ also belong to $B_{k}$. There are $m ! b_{1} ! \cdots b_{m}$ ! such consistent orderings. Assume that each ordering is equally likely. The CS value of the game $(I, V)$ is a rule which assigns to each agent $i$ the expected marginal contribution to every coalition that agent $i$ is a member of, respecting the coalition structure $\mathcal{B}$. That is, agent $i$ 's expected marginal contribution is given by

$$
\phi_{i}(V, \mathcal{B})=E\left[V\left(P^{i} \cup\{i\}\right)-V\left(P^{i}\right)\right],
$$

where the expectation is over all random orders on $I$ that are consistent with $\mathcal{B}$, and $P^{i}$ denotes the set of random predecessors of player $i$.

For example, if no agent forms a coalition with another agent, then the coalition structure $\mathcal{B}=\{\{1\}, \ldots,\{n\}\}$. In this case the CS Shapley value (5) value coincides with the standard Shapley value (4).

As another example, assume that $I=\{1,2,3\}$, and $\mathcal{B}=\{\{1,2\},\{3\}\}$. Then there are the following consistent orderings

$$
1 \prec 2 \prec 3 \quad 2 \prec 1 \prec 3 \quad 3 \prec 1 \prec 2 \quad 3 \prec 2 \prec 1 .
$$

Therefore,

$$
\begin{aligned}
\phi_{3}(V, \mathcal{B})= & \frac{1}{4}(V(\{1,2,3\})-V(\{1,2\}))+\frac{1}{4}(V(\{2,1,3\})-V(\{2,1\}))+\frac{1}{4}(V(\{3\}) \\
& -V(\emptyset))+\frac{1}{4}(V(\{3\})-V(\emptyset))=\frac{1}{2}(V(\{1,2,3\})-V(\{1,2\}))+\frac{1}{2} V(\{3\}) .
\end{aligned}
$$

Agent 3's CS Shapley value is therefore the same as the standard value of a game where we treat agents 1 and 2 as a single player. The insight that coalitions of agents in the CS value can be treated as single agents in the standard Shapley value is true in general (see Corollary 2.4 in Hart and Kurz, 1983). The CS Shapley value therefore measures agents' expected contributions after coalitions have formed for the purpose of bargaining. It is also important to note that the CS Shapley value is Pareto efficient, i.e. $\sum_{i \in I} \pi_{i}(V, \mathcal{B})=V(I)$. Quoting Hart and Kurz (1983), "the efficiency of the CS value is an essential feature. It differs from the Aumann and Dreze (1974) approach, where each coalition $B_{k} \in B$ gets only its worth (i.e. $V\left(B_{k}\right)$ ). The idea is that coalitions form not in order to get their worth, but to be in a better position when bargaining with the others on how to divide the maximal amount available."

We now describe the notion of a CS value allocation for an economy with private information. These concepts generalize those of Shapley value allocation introduced in Krasa and Yannelis (1994).

In (2) and (3) of Section 3 we have introduced two alternative ways to assign a TU game to an economy with differential information. In (2) agents' trades are required to be measurable with respect to private information. In (3) we replace private measurability with incentive compatibility. As in Krasa and Yannelis (1994) we therefore get two alternative definitions of a Shapley value allocation. Note that if $\mathcal{B}$ is replaced by the fine partition, then the following two concepts coincide with those of Krasa and Yannelis (1994). 
Definition 3. An allocation $\left(x_{i}\right)_{i \in I}$ is a private CS value allocation of the economy with differential information, $\mathcal{E}$, if the following holds:

(i) $x_{i}$ is $\mathcal{F}_{i}$-measurable for all $i \in I$.

(ii) $\sum_{i=1}^{n} x_{i}(\omega)=\sum_{i=1}^{n} e_{i}(\omega)$, $\mu$-a.e.

(iii) There exist $\lambda_{i} \geq 0$, for every $i=1, \ldots, n$, which are not all equal to zero, with $\lambda_{i} \int u_{i}\left(x_{i}(\omega)\right) \mathrm{d} \mu(\omega)=\phi_{i}\left(V_{\lambda}^{p}, \mathcal{B}\right)$, where $\phi_{i}\left(V_{\lambda}^{p}, \mathcal{B}\right)$ is the CS Shapley value of agent $i$ derived from the game $\left(I, V_{\lambda}^{p}\right)$, defined in (2).

Definition 4. An allocation $\left(x_{i}\right)_{i \in I}$ is a Bayesian incentive compatible (BIC) CS value allocation of the economy with differential information, $\mathcal{E}$, if the following holds:

(i) $x_{i}$ is $\bigvee_{i \in I} \mathcal{F}_{i}$-measurable for all $i \in I$, and $\left(x_{i}\right)_{i \in I}$ is BIC for coalition $I$.

(ii) $\sum_{i=1}^{n} x_{i}(\omega)=\sum_{i=1}^{n} e_{i}(\omega)$, $\mu$-a.e.

(iii) There exist $\lambda_{i} \geq 0$, for every $i=1, \ldots, n$, which are not all equal to zero, with $\lambda_{i} \int u_{i}\left(x_{i}(\omega)\right) \mathrm{d} \mu(\omega)=\phi_{i}\left(V_{\lambda}^{\mathrm{BIC}}, \mathcal{B}\right)$, where $\phi_{i}\left(V_{\lambda}^{\mathrm{BIC}}, \mathcal{B}\right)$ is the CS Shapley value of agent $i$ derived from the game $\left(I, V_{\lambda}^{\mathrm{BIC}}\right)$, defined in (3).

Note that the existence proof of Shapley (1953) can be easily modified to cover the coalition structure value. Given this result one can adapt the existence proof in Krasa and Yannelis (1996) to show that a private coalition structure value exists. Theorem 1 then immediately implies that the private CS value allocation is Bayesian incentive compatible. We now state this result formally.

Theorem 2. The private CS value allocation of a differential information economy exists and is BIC for the grand coalition I.

In contrast to the private CS value allocation, the BIC CS value allocation need not always exist because the set of feasible incentive compatible allocations need not be convex. In the economy of Section 5 the two concepts coincide. Hence, our conclusions about the advantages or disadvantages of coalitional bargaining hold for both concepts.

\section{Is unity a strength?}

We now consider an economy with three agents $i=1,2,3$. In this economy agents 1 and 2 seek insurance against their uncertain endowment realization. Agents 1 and 2 can insure each other, but they can also seek insurance from agent 3 who is risk neutral.

First consider the case of complete information, i.e. once the state is realized it becomes public information. As one would expect, agents 1 and 2 are better off if they collectively bargain against agent 3 . That is, if information is complete the CS Shapley value for agents 1 and 2 is strictly higher than the standard Shapley value. However, once asymmetric information is introduced the result is completely reversed. Now agents 1 and 2 are strictly better off if they do not form a coalition. In this sense, "unity is strength" does not hold when information is asymmetric. 
Assume there are four states $\omega \in \Omega=\{a, b, c, d\}$ that are equally likely. There is one consumption good in each state. The agents' utility functions are given by

$$
\begin{aligned}
& u_{1}(x, \omega)=\left\{\begin{array}{ll}
x & \text { if } \omega=a, b, d, \\
2 \sqrt{x} & \text { if } \omega=c,
\end{array} \quad u_{2}(x, \omega)= \begin{cases}x & \text { if } \omega=a, c, d, \\
2 \sqrt{x} & \text { if } \omega=b,\end{cases} \right. \\
& u_{3}(x, \omega)=x, \forall \omega .
\end{aligned}
$$

The agents' endowments are

$$
\begin{aligned}
& e_{1}(\omega)=\left\{\begin{array}{ll}
2 & \text { if } \omega=a, b, \\
0 & \text { if } \omega=c, d,
\end{array} \quad e_{2}(\omega)= \begin{cases}2 & \text { if } \omega=a, c, \\
0 & \text { if } \omega=b, d,\end{cases} \right. \\
& e_{3}(\omega)= \begin{cases}\frac{1}{4} & \text { if } \omega=a, b, c \\
10 & \text { if } \omega=d .\end{cases}
\end{aligned}
$$

Agents 1 and 2 are risk averse in states $c$ and $b$, respectively. The agents therefore seek insurance against the low endowment realization in these states. Note that the role of state $d$ is to ensure that the weights $\lambda_{i}$ in the TU game are all equal.

In order to derive the value allocation, we must find the utility weights $\lambda_{i}, i=1,2,3$. However, because agents' utilities are quasilinear all weights $\lambda_{i}$ must be equal, i.e. $\lambda_{1}=$ $\lambda_{2}=\lambda_{3}=1$. This is shown formally in Lemma 1 in Appendix A. Therefore, the economy corresponds to a game with transferable utility and we can write $V(S)$ instead of $V_{\lambda}(S)$ to denote the payoff of coalition $S$.

Complete information: Assume that all agents learn the true state $\omega$ after it is realized. For any coalition $S$, let $V(S)$ be the maximum attainable utility, i.e.

$$
V(S)=\max _{\left\{x_{i}(\omega) \mid i \in S, \omega \in \Omega\right\}} \sum_{i \in S} \sum_{\omega \in \Omega} \frac{1}{4} u_{i}\left(\omega, x_{i}(\omega)\right) \text { s.t. } \quad \sum_{i \in S} x_{i}(\omega)=\sum_{i \in S} e_{i}(\omega), \quad \forall \omega \in \Omega .
$$

For example, assume that agent 1 belongs to $S$. Then it follows immediately that it is optimal to choose $x_{1}(c)=1$. The same is true for agent 2 and state $b$.

The TU game is therefore given by

$$
\begin{aligned}
& V(\{1\})=V(\{2\})=1, V(\{3\})=\frac{43}{16}, \quad V(\{1,2\})=\frac{5}{2}, V(\{1,3\})=V(\{2,3\})=\frac{31}{8}, \\
& V(\{1,2,3\})=\frac{83}{16} .
\end{aligned}
$$

The resulting Shapley values are

$$
\mathrm{Sh}_{1}=\mathrm{Sh}_{2}=\frac{39}{32}, \quad \mathrm{Sh}_{3}=\frac{11}{4} \text {. }
$$

A Shapley value allocation is given by

$$
x_{1}=x_{2}=\left(1,1,1, \frac{7}{8}\right), \quad x_{3}=\left(\frac{9}{4}, \frac{1}{4}, \frac{1}{4}, \frac{33}{3}\right) .
$$

Now assume agents 1 and 2 form a coalition. Then by Corollary 2.4 in Hart and Kurz (1983), agent 3's Shapley value can be computed from the Shapley value of the game where $\{1,2\}$ 
is treated as a single player. Moreover, because of symmetry the Shapley values of agents 1 and 2 must be exactly one half of the Shapley value of the "single player" $\{1,2\}$. Then

$$
\phi_{1}(\mathcal{B})=\phi_{2}(\mathcal{B})=\frac{5}{4}, \quad \phi_{3}(\mathcal{B})=\frac{43}{16} .
$$

A CS Shapley value allocation is given by

$$
x_{1}=x_{2}=(1,1,1,1), \quad x_{3}=\left(\frac{9}{4}, \frac{1}{4}, \frac{1}{4}, 8\right) .
$$

Therefore, $\phi_{3}(\mathcal{B})<\mathrm{Sh}_{3}$ and $\phi_{i}(\mathcal{B})>\mathrm{Sh}_{i}$, for $i=1$, 2, i.e. agents 1 and 2 are strictly better off when they bargain together. Hence, "unity is strength" in this case.

Differential information: Assume that agent 1 cannot distinguish states $a$ and $b$, and that agent 2 cannot distinguish states $a$ and $c$. Agent 3 has complete information. ${ }^{3}$ Again, the economy corresponds to a TU game. The payoffs in the TU game are the same as above except that

$$
V^{p}(\{1,2\})=V^{\mathrm{BIC}}(\{1,2\})=2 .
$$

We first show that all coalitions $S \neq\{1,2\}$ have the same payoff under differential information as under complete information. This is obvious for all single agent coalitions. Next, note that (7) indicates an allocation $\left(x_{i}\right)_{i \in I}$, for which each $x_{i}$ is $\mathcal{F}_{i}$-measurable, and which yields the payoff $V(I)$. Theorem 1 implies that $\left(x_{i}\right)_{i \in I}$ is BIC for coalition $I$. Therefore, $V^{p}(I)=V^{\mathrm{BIC}}(I)=V(I)$. Next, consider coalition $S=\{1,3\}$. The payoff $V(\{1,3\})$ can be obtained by choosing $x_{1}=(1,1,1 / 4,0)$ and $x_{3}=(5 / 4,5 / 4,0,10)$. This allocation for coalition $S$ is measurable with respect to each agent's private information and therefore also BIC. The argument for $S=\{2,3\}$ is similar.

Finally, consider coalition $S=\{1,2\}$. The only feasible allocation $\left(x_{i}\right)_{i \in S}$ that is measurable with respect to private information consists of the agents' endowments. In particular feasibility implies $x_{1}-e_{1}=x_{2}-e_{2}$. Private measurability implies that $x_{1}-e_{1}$ is $\mathcal{F}_{1}$-measurable and that $x_{2}-e_{2}$ is $\mathcal{F}_{2}$-measurable. Therefore, each $x_{i}-e_{i}$ must be $\mathcal{F}_{1} \wedge \mathcal{F}_{2}$-measurable. Since $\mathcal{F}_{1} \wedge \mathcal{F}_{2}=\{\{a, b, c\},\{d\}\}$ the only feasible trades are trivial. It is also not possible to improve upon autarky via BIC net trades. In order to improve upon autarky, either $x_{1}(c)$ or $x_{2}(b)$ must be strictly positive. Let $\omega^{*}=a$ and assume that agent 1 reports $\omega^{\prime}=c$. Then $Z_{S}\left(\omega^{*}\right)=\{a\}$. Therefore, incentive compatibility implies $x_{1}(a) \geq 2+x_{1}(c)$. Similarly, $x_{2}(a) \geq 2+x_{2}(b)$ must hold. Therefore, $x_{1}(c)+x_{2}(b) \leq 0$ which implies $x_{1}(c)=x_{2}(b)=0$.

The Shapley values are therefore given by

$$
\mathrm{Sh}_{1}=\mathrm{Sh}_{2}=\frac{109}{96}, \quad \mathrm{Sh}_{3}=\frac{35}{12} \text {. }
$$

A private or BIC Shapley value allocation is given by

$$
x_{1}=x_{2}=\left(1,1,1, \frac{13}{24}\right), \quad x_{3}=\left(\frac{9}{4}, \frac{1}{4}, \frac{1}{4}, \frac{107}{12}\right) .
$$

Now assume that agents 1 and 2 form a coalition. Then

$$
\phi_{1}(\mathcal{B})=\phi_{2}(\mathcal{B})=\frac{9}{8}, \quad \phi_{3}(\mathcal{B})=\frac{47}{16} .
$$

\footnotetext{
$\overline{{ }^{3} \text { Formally }}, \mathcal{F}_{1}=\{\{a, b\},\{c\},\{d\}\}, \mathcal{F}_{2}=\{\{a, c\},\{b\},\{d\}\}$, and $\mathcal{F}_{3}=\{\{a\},\{b\},\{c\},\{d\}\}$.
} 
A BIC or a private CS Shapley value allocation is given by

$$
x_{1}=x_{2}=\left(1,1,1, \frac{1}{2}\right), \quad x_{3}=\left(\frac{9}{4}, \frac{1}{4}, \frac{1}{4}, 9\right) .
$$

Therefore, $\phi_{3}(\mathcal{B})>\mathrm{Sh}_{3}$ and $\phi_{i}(\mathcal{B})<\mathrm{Sh}_{i}$, for $i=1,2$. As a consequence, when information is incomplete agents 1 and 2 are strictly worse off it they bargain as a coalition. Therefore, in this case unity among coalition members is not a strength.

\subsection{Interpretation}

Although there is no explicit time structure in our model, Eq. (6), and more generally Corollary 2.4 in Hart and Kurz (1983), show that we can interpret the CS value as follows.

First, agents 1 and 2 decide to form a coalition. They sign an agreement that determines how any surplus will be split among them. After the agreement is signed, coalition $\{1,2\}$ bargains with agent 3 . Because agents 1 and 2 can provide insurance to each other without the help of an outside agent, agent 3's service is not essential. Coalition $\{1,2\}$ is therefore in the strongest possible bargaining position, and agent 3 will receive only a small percentage of the surplus. In contrast, when agent 1 or 2 bargains separately with agent 3 , then agent 3 can provide a significant service by insuring that agent. As a consequence, agent 3 is able to extract a higher percentage of the surplus when agents 1 and 2 bargain separately.

As another example, consider a coalition of firms that bargains with an outside firm for a service. If the coalition is sufficiently diverse, it is likely that the outsider's service could be replaced by that of a coalition member, and is therefore not that essential for the coalition. As a consequence, the outside firm will find itself in a weak bargaining position. Unity among coalition members is therefore a strength.

What goes wrong with this intuition when information is asymmetric? In our example, agent 3 has information that is essential for agents 1 and 2 . The value of agent 3 's information increases with the number of agents that need agent 3's information. Formally, $V(\{1,2,3\})-$ $V(\{3\})>V(\{1,3\})-V(\{3\})=V(\{2,3\})-V(\{3\})$. In other words, the information becomes more important the larger the coalition of agents that depends on it. Therefore, ceteris paribus, agent 3 will extract a high percentage of the surplus if he only bargains with large coalitions.

Consider again the example of the coalition of firms, but now assume that the outside firm has information that is crucial to the coalition. Then the outside firm will be able to sell its information for a higher price (i.e. a higher percentage of the surplus) the larger the coalition. Intuitively, the outside firm can hold the coalition "hostage" to its information. "unity is a strength" may therefore cease to be true when informational asymmetries matter.

\section{Acknowledgements}

Temimi acknowledges financial support from the Culverhouse College of Commerce and Business Administration at the University of Alabama. 


\section{Appendix A}

Proof of Theorem 1. Because statement 2 implies statement 1, it is sufficient to prove 2 . Assume by contradiction that $\left(x_{i}\right)_{i \in S}$ is feasible for coalition $S$, but not BIC for coalition $S$.

Then there exists an agent $j \in S$, an $\omega^{*}, \omega^{\prime}$ such that

$$
\begin{gathered}
\int_{Z_{S}\left(\omega^{*}\right)} u_{j}\left(\omega, e_{j}(\omega)-\left(x_{j}\left(\omega^{\prime}\right)-e_{j}\left(\omega^{\prime}\right)\right)\right) \mathrm{d} \mu\left(\omega \mid E_{j}\left(\omega^{*}\right)\right) \\
>\int_{Z_{S}\left(\omega^{*}\right)} u_{j}\left(\omega, x_{j}(\omega)\right) \mathrm{d} \mu\left(\omega \mid E_{j}\left(\omega^{*}\right)\right),
\end{gathered}
$$

where $\omega^{\prime} \in \bigcap_{i \in S \backslash\{j\}} E_{i}\left(\omega^{*}\right)$ and $Z_{S}\left(\omega^{*}\right)=\bigcap_{i \in S} E_{i}\left(\omega^{*}\right)$. For the fixed $\omega^{*} \in \Omega$, define $y_{i}: \Omega \rightarrow \mathbb{R}_{+}^{\ell}$ for each agent $i$ by

$$
y_{i}(\omega)= \begin{cases}e_{i}(\omega)+x_{i}\left(\omega^{\prime}\right)-e_{i}\left(\omega^{\prime}\right) & \text { for } \omega \in Z_{S}\left(\omega^{*}\right), \\ x_{i}(\omega) & \text { for } \omega \notin Z_{S}\left(\omega^{*}\right) .\end{cases}
$$

It can be easily checked that $\sum_{i \in S} y_{i}(\omega)=\sum_{i \in S} e_{i}(\omega), \mu$-a.e.

Consider an agent $i \in S \backslash\{j\}$. Let $\omega \in Z_{S}\left(\omega^{*}\right)$. Then $\omega^{\prime} \in \bigcap_{k \in S \backslash\{j\}} E_{k}\left(\omega^{*}\right) \subset$ $E_{i}\left(\omega^{*}\right)$ and $Z_{S}\left(\omega^{*}\right) \subset E_{i}\left(\omega^{*}\right)$ imply that agent $i$ cannot distinguish $\omega$ from $\omega^{\prime}$. Therefore, $\mathcal{F}_{i}$-measurability of $x_{i}$ implies that $x_{i}(\omega)=x_{i}\left(\omega^{\prime}\right)$ for all $\omega \in Z_{S}\left(\omega^{*}\right)$. Thus, $y_{i}=x_{i}$ for all $i \in S \backslash\{j\}$. This and feasibility imply

$$
y_{j}=\sum_{i \in S} e_{i}-\sum_{i \in I \backslash\{j\}} y_{i}=\sum_{i \in S} e_{i}-\sum_{i \in I \backslash\{j\}} x_{i}=x_{j} .
$$

Eq. (11) immediately implies

$$
\int_{Z_{S}\left(\omega^{*}\right)} u_{j}\left(\omega, y_{j}(\omega)\right) \mathrm{d} \mu\left(\omega \mid E_{j}\left(\omega^{*}\right)\right)=\int_{Z_{S}\left(\omega^{*}\right)} u_{j}\left(\omega, x_{j}(\omega)\right) \mathrm{d} \mu\left(\omega \mid E_{j}\left(\omega^{*}\right)\right) .
$$

However, in view of (10), the left-hand side of (12) is equal to (9). Therefore, (12) contradicts (9). Consequently, $\left(x_{i}\right)_{i \in S}$ is BIC for coalition $S$.

Lemma 1. Consider the exchange economies of Section 5. Then all utility weights are identical in all coalition structure and in all standard Shapley value allocations both with complete and incomplete information.

Proof. Assume by way of contradiction that the utility weights differ. In the case of complete information, the agents' consumption in the Shapley value allocation must solve

$$
\max _{\left\{x_{i}(\omega) \mid i \in I, \omega \in \Omega\right\}} \sum_{i \in I} \sum_{\omega \in \Omega} \frac{1}{4} \lambda_{i} u_{i}\left(\omega, x_{i}(\omega)\right) \quad \text { s.t. } \quad \text { (i) } \sum_{i \in I} x_{i}(\omega)=\sum_{i \in I} e_{i}(\omega), \quad \forall \omega \in \Omega .
$$

With differential information we must add one of the following constraints.

(iia) $x_{i}$ is $\mathrm{BIC} \forall i \in I$.

(iib) $x_{i}$ is $\mathcal{F}_{i}$-measurable $\forall i \in I$. 
It follows immediately, that (iia) is always slack, and can therefore be omitted. In particular, because agent 3 has complete information, any misreport by agent 1 or agent 2 is immediately detected, i.e. the sets $\bigcap_{j \in I \backslash\{i\}} E_{j}\left(\omega^{*}\right)$ are singletons for $i=1$, 2, which implies $\omega^{*}=\omega^{\prime}$. Moreover, because $\mathcal{F}_{1} \vee \mathcal{F}_{2}=\mathcal{F}_{3}$, agent 3 can also not misreport his information, i.e. $E_{1}\left(\omega^{*}\right) \cap E_{2}\left(\omega^{*}\right)$ only contains $\omega^{*}$.

We now prove by way of contradiction that all weights $\lambda_{i}$ are identical. It should be noted that is does not matter in the argument whether or not (iib) is imposed.

First, assume that $\lambda_{1}<\lambda_{3}$. Then agent 1's consumption in states $a, b$, and $d$ must be zero. Therefore, agent 1's expected utility is $\mu(\{c\}) 2 \sqrt{x_{3}(c)}$. Because of feasibility $x_{3}(c) \leq 9 / 4$. Individual rationality is therefore violated for agent 1 . Thus, $\lambda_{1} \geq \lambda_{3}$. A similar argument shows that $\lambda_{2} \geq \lambda_{3}$.

Now assume that $\lambda_{3}<\lambda_{1}$ or $\lambda_{3}<\lambda_{2}$. Then $x_{3}(d)=0$. However, because of feasibility, $x_{3}(a) \leq 4$ and $x_{3}(b), x_{3}(c) \leq 2$. Therefore individual rationality would be violated for agent 3. This implies $\lambda_{1}=\lambda_{2}=\lambda_{3}$.

\section{References}

Allen, B., Yannelis, N.C., 2001. Differential information economies: introduction. Economic Theory 18, $263-273$. Aumann, R.J., Dreze, J., 1974. Cooperative games with coalition structures. International Journal of Game Theory 3, 214-237.

Glycopantis, D., Muir, A., Yannelis, N.C., 2001. An extensive form interpretation of the private core. Economic Theory 18, 293-319.

Hart, S., Kurz, M., 1983. Endogenous formation of coalitions. Econometrica 51, 1047-1064.

Krasa, S., Yannelis, N.C., 1994. The value allocation of an economy with differential information. Econometrica 62, 881-900.

Krasa, S., Yannelis, N.C., 1996. Existence and properties of a value allocation for an economy with differential information. Journal of Mathematical Economics 25, 165-179.

Owen, G., 1977. Values of games with a priori unions. In: Essays in Mathematical Economics and Game Theory. Springer Verlag, Berlin.

Shapley, L.S., 1953. A value for $n$-person games. In: Contributions to the Theory of Games. Princeton University Press, Princeton, NJ. 